\title{
Static and Dynamical Properties of the Ferromagnetic Kondo Model with Direct Antiferromagnetic Coupling Between the localized $t_{2 g}$ Electrons
}

\author{
Seiji Yunoki and Adriana Moreo \\ Department of Physics, National High Magnetic Field Lab and MARTECH, Florida State University, Tallahassee, FL 32306, \\ USA
}

(December 3, 2017)

\begin{abstract}
The phase diagram of the Kondo lattice Hamiltonian with ferromagnetic Hund's coupling in the limit where the spin of the localized $t_{2 g}$ electrons is classical is analyzed in one dimension as a function of temperature, electronic density, and a direct antiferromagnetic coupling $J^{\prime}$ between the localized spins. Studying static and dynamical properties, a behavior that qualitatively resembles experimental results for manganites occurs for $J^{\prime}$ smaller than 0.11 in units of the $e_{g}$ hopping amplitude. In particular a coexistence of ferromagnetic and antiferromagnetic excitations is observed at low-hole density in agreement with neutron scattering experiments on $\mathrm{La}_{2-2 x} \mathrm{Sr}_{1+2 \mathrm{x}} \mathrm{Mn}_{2} \mathrm{O}_{7}$ with $x=0.4$. This effect is caused by the recently reported tendency to phase separation between holerich ferromagnetic and hole-undoped antiferromagnetic domains in electronic models for manganites. As $J^{\prime}$ increases metal-insulator transitions are detected by monitoring the optical conductivity and the density of states. The magnetic correlations reveal the existence of spiral phases without longrange order but with fairly large correlation lengths. Indications of charge ordering effects appear in the analysis of charge correlations.
\end{abstract}

PACS numbers: 771.10.-w, 75.10.-b, 75.30.Kz

\section{INTRODUCTION}

The study of manganese oxides with perovskite-type structure such as $\mathrm{La}_{1-\mathrm{x}} \mathrm{Ca}_{\mathrm{x}} \mathrm{MnO}_{3}$ and $\mathrm{La}_{1-\mathrm{x}} \mathrm{Sr}_{\mathrm{x}} \mathrm{MnO}_{3}$ has received new emphasis since the discovery of colossal magnetoresistance in these compounds [1]. Experiments have revealed a rich phase diagram with antiferromagnetic insulating, ferromagnetic metallic, and charge ordered regions [2,3]. Recently, layered compounds with properties similar to the $3 \mathrm{D}$ materials have been synthesized: the new compounds are described by the general formula $(\mathrm{A}, \mathrm{B})_{\mathrm{n}+1} \mathrm{Mn}_{\mathrm{n}} \mathrm{O}_{3 \mathrm{n}+1}$, where $n$ is the number of $\mathrm{Mn}-\mathrm{O}$ planes per unit cell $(n=\infty$ corresponds to the 3D case) [4]. The appearance of ferromagnetism upon hole doping away from $x=0$ has been attributed to the double-exchange (DE) mechanism between the $e_{g}$ and $t_{2 g}$ electrons [5]. However, this model is not sufficient to describe the rich phase diagram observed experimentally. The large regions in the phase diagram which present charge ordering are not contained in the DE model. Features such as the antiferromagnetic phase in the low $e_{g}$-electronic density limit $(x \sim 1)$, or the related experimentally observed antiferromagnetic ground state detected in $\mathrm{La}_{1-\mathrm{x}} \mathrm{Ca}_{\mathrm{x}} \mathrm{MnO}_{3}$ for $x>0.55$ are difficult to explain by DE ideas. The Néel temperature of this state reaches a maximum value of $260 \mathrm{~K}$ and decreases to $120 \mathrm{~K}$ at $x=1$ [2].

The main goal of this paper will be to study the phase diagram of a modified ferromagnetic (FM) Kondo lattice Hamiltonian in order to understand qualitatively what properties of the manganites are solely due to electronic interactions. An analysis including Jahn-Teller phonons [8] will be postponed for a future publication. To achieve our goal the ferromagnetic Kondo lattice Hamiltonian will be here modified by introducing a direct antiferromagnetic exchange interaction, $J^{\prime}$, between the spins of the localized $t_{2 g}$ electrons [9, 10]. This coupling will certainly produce an antiferromagnetic phase at $x=1$, as experimentally observed, which does not appear in the standard FM Kondo model. The addition of this coupling is certainly not unphysical since it may originate from small hopping amplitudes for the $t_{2 g}$ electrons. Experimentally such an effective antiferromagnetic exchange interaction $J^{\prime}$ has been studied in the $2 \mathrm{D}$ compounds and $2 J^{\prime} S \approx 100 K$ has been estimated 11].

Among the main issues that will be addressed in the present manuscript is the influence of the direct coupling $J^{\prime}$ among the $t_{2 g}$ electrons on the recently reported regime of phase separation detected in the ferromagnetic Kondo model [10]. This effect, as it will be discussed later, could be a key feature to understand some of the recent neutron scattering results for the manganites.

The organization of the paper is the following: in Section II the Hamiltonian will be introduced. Phase separation will be studied in Section III. Magnetic and charge ordering will be discussed in Section IV. In Section V the existence of a Fermi surface is studied by monitoring $n(k)$ and the spectral function $A(k, \omega)$. The optical conductivity, the Drude weight and metal-insulator transitions are presented in Section VI. In Section VII the phase diagram is proposed. Section VIII is devoted to summarizing the results. 


\section{THE MODEL}

The parent compound $\mathrm{LaMnO}_{3}$ contains $\mathrm{Mn}^{3+}$ ions in a $t_{2 g}^{3} e_{g}^{1}$ configuration. The $t_{2 g}^{3}$ electrons may be viewed as a localized $S=3 / 2$ spin, while the $e_{g}^{1}$ electrons are mobile. Here one single orbital for the $e_{g}$ electrons will be considered. To describe the doped manganites a Kondo lattice model with ferromagnetic Hund coupling has been proposed 12,13. In spite of its apparent simplicity, this model is still difficult to study accurately. In order to simplify its analysis the localized $S=3 / 2$ spins will be replaced by classical spins with $\left|\mathbf{S}_{i}\right|=1$ 14. In addition, an antiferromagnetic coupling $J^{\prime}$ will be added between the classical spins to obtain the antiferromagnetic phase at $x=1$ as explained in the Introduction [9, 10]. Finally, since the standard FM Kondo Hamiltonian in 3D is difficult to study numerically due to its large number of degrees of freedom, some other simplifications must be introduced. Since previous studies have convincingly shown that the basic features of the phase diagram in three dimensional problems appear in two and one dimensions as well [10], results in one dimension will be here presented. A careful study of the $1 \mathrm{D}$ problem in chains with up to 40 sites will be carried out using Monte Carlo (MC) techniques. by

Then, the Hamiltonian studied in this paper is given

$$
\begin{gathered}
H=-t \sum_{\langle i j\rangle, \alpha}\left(c_{i, \alpha}^{\dagger} c_{j, \alpha}+h . c .\right) \\
-J_{H} \sum_{i, \alpha, \beta} c_{i, \alpha}^{\dagger} \sigma_{\alpha, \beta} c_{i, \beta} \cdot \mathbf{S}_{i}+J^{\prime} \sum_{\langle i j\rangle} \mathbf{S}_{i} \cdot \mathbf{S}_{j}
\end{gathered}
$$

where $c_{i, \alpha}^{\dagger}$ creates an $e_{g}$ electron at site $i$ with spin projection $\alpha, \sigma$ are Pauli matrices, $\mathbf{S}_{i}$ is the total spin of the $t_{2 g}$ electrons assumed localized and classical, the sum $\langle i j\rangle$ runs over pairs of nearest-neighbor lattice sites, $t$ is the nearest-neighbor hopping amplitude for the $e_{g}$ electrons, $J_{H}>0$ is the Hund coupling, and $J^{\prime}>0$ is an antiferromagnetic coupling between the localized spins. The density $\langle n\rangle=1-x$ of itinerant $e_{g}$-electrons is controlled by a chemical potential $\mu$. In the following $t=1$ will be used as the unit of energy and $J_{H}$ will be fixed at 8 since phenomenologically $J_{H} \gg t$. Periodic boundary conditions (PBC) will be used. The model will be studied with the Monte Carlo technique already described in Ref. [10]. This method basically amounts to integrating exactly the $e_{g}$ electrons using library subroutines (since it corresponds to a one-electron problem in the background of the localized spins), supplemented by a Monte Carlo simulation on the $t_{2 g}$ classical spins. The technique does not have "sign" problems and it allows to reach low temperatures at any density. The neglect of the direct Coulomb interactions among the $e_{g}$ electrons can be justified recalling that a large Hund coupling effectively reduces double occupancy, similarly as a large on-site Hubbard repulsion does.

\section{PHASE SEPARATION}

In Ref. 10 the phase diagram of model Eq.(1) has been studied for the special case $J^{\prime}=0$. In that paper it was found that at low temperatures the antiferromagnetic insulating phase that appears at $x=0$ is phase separated from a metallic ferromagnetic phase that becomes stable for $x>0.25$. Here the effect that a finite $J^{\prime}$ has on this important result will be investigated.

To study phase separation the density $\langle n\rangle$ as a function of the chemical potential $\mu$ has to be monitored. Working with a chain of $L$ sites and at zero temperature $(T)$, the density $\langle n\rangle$ can take only the values $\frac{m}{L}$ with $m$ running from 0 to $2 L$. However, at finite $T$ the effects of thermal fluctuations allow for intermediate values of $\langle n\rangle$ to exist, and this complicates the study of phase separation since sharp discontinuities are usually transformed into rapid crossovers. This behavior is clearly observed in long MC runs when tunneling between several of the allowed states occurs. In particular during MC runs of 40,000 iterations at low temperature the system was observed to tunnel several times between states with a sharply defined value of $\langle n\rangle$ for particular values of the chemical potential. In Fig. 1 results for a Monte Carlo run for $\mu=-6.97$ on an $L=20$ chain and at $T=1 / 75$ are presented as an illustration. The density clearly fluctuates between states with 15, 16, 17, 18 and 19 electrons. This particular behavior allowed us to measure ground state properties for most of the possible filling levels by simply carrying out measurements of, e.g., the density and energy in the plateaus observed in Fig.1. With this information (i.e. energy vs density without temperature contaminations) it was possible to perform an accurate analytical calculation of $\langle n\rangle$ vs $\mu$ at $T=0$ using the Maxwell construction. This method allows us to obtain ground state information from a finite temperature simulation.

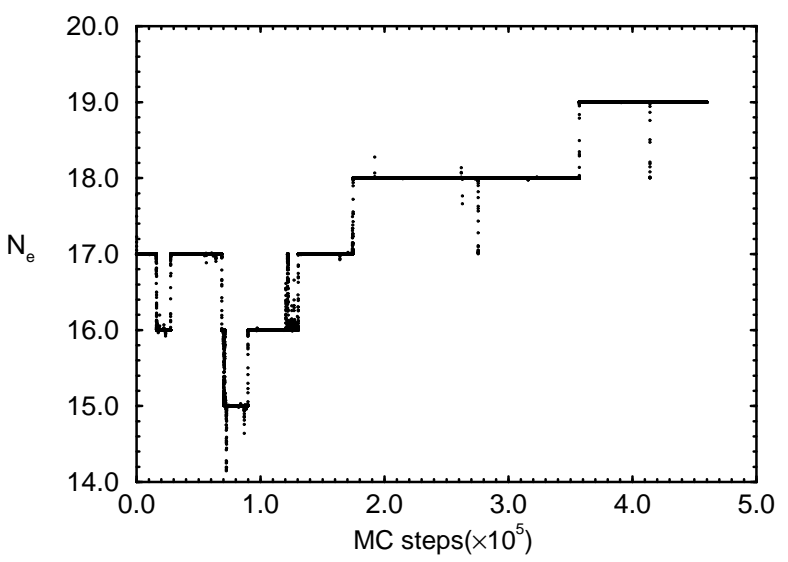

FIG. 1. The total number of itinerant electrons $N_{e}$ as a function of the Monte Carlo steps for an $L=20$ chain with $J_{H}=8, J^{\prime}=0.05, T=1 / 75$ and $\mu=-6.97$. 
In Fig.2a the relation $\langle n\rangle$ vs $\mu$ at $T=0$ obtained analytically from the MC results at $J^{\prime}=0.05$ on an $L=20$ lattice (solid line) is presented, and it is compared with the results obtained directly from Monte Carlo simulations at $T=0.01$ (open circles) [15]. The $T=0$ curve shows that one regime of phase separation (denoted by PS1) occurs between regions with $\langle n\rangle=1$ (i.e., $x=0$ ) and $\langle n\rangle=0.65$. This behavior is similar to the results recently reported in Ref. [10] working at $J^{\prime}=0$. However, the same figure shows the novel result that there is a second region of phase separation (denoted by PS2) in the limit of low $e_{g}$-electronic density between the empty system $\langle n\rangle=0.0$ and an electron-rich phase with $\langle n\rangle=0.25$. Through a careful analysis it has been found that phase separation at low density also occurs for smaller values of $J^{\prime}$ such as 0.02 .
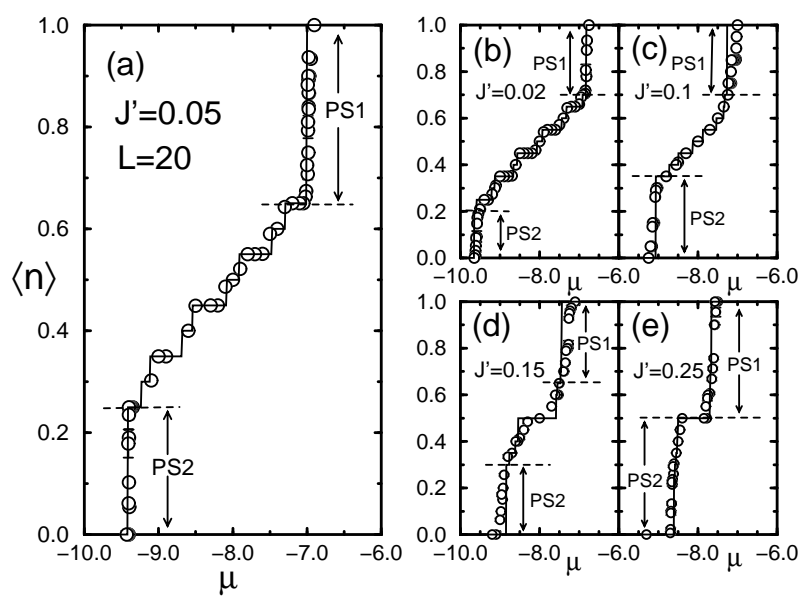

FIG. 2. The density of itinerant electrons $\langle n\rangle$ as a function of the chemical potential $\mu$ on $L=20$ chains with $J_{H}=8$ and (a) $J^{\prime}=0.05$, (b) $J^{\prime}=0.02$, (c) $J^{\prime}=0.1$, (d) $J^{\prime}=0.15$ and (e) $J^{\prime}=0.25$. The open circles indicate Monte Carlo results at $T=0.01$. The lines are obtained using the Maxwell construction and they indicate $T=0$ behavior.

It is important to remark that at $J^{\prime}=0$ indications of a rapid crossover at low densities exist but this effect does not reach the full character of phase separation since at $x=1$ the $t_{2 g}$ spins are non-interacting in this special case. In Fig. $2 \mathrm{~b}-\mathrm{e},\langle n\rangle$ vs $\mu$ curves at $T=0$ for different values of $J^{\prime}$ using chains with $L=20$ sites are presented (obtained using the methods discussed above that allowed us to remove the temperature contaminations from the results). Additional MC runs using open boundary conditions and different lattice sizes close to the phase separation critical chemical potentials have shown that the results are not affected by strong finite size effects, and thus we are confident that they already represent the bulk limit. In general, it was observed (Fig.2) that as $J^{\prime}$ increases the range of stable densities decreases, and for $J^{\prime} \geq 0.25$ the only stable density besides $\langle n\rangle=1$ and 0 is $\langle n\rangle=0.5$. Evenmore, for values of $J^{\prime}$ larger than 0.6 only the empty and the half-filled phases are stable. This remarkably strong tendency to phase separation can be qualitatively understood in the limit where $J^{\prime}$ is larger than $t$, both still smaller than the Hund coupling. In this regime the antiferromagnetic order caused by $J^{\prime}$ among the localized spins induces a similar antiferromagnetic tendency among the itinerant electrons (again, note that the Hund coupling is assumed to be the largest scale). Then, in the conduction band an effective one band $t-J^{\prime}$ model with $J^{\prime} / t \gg 1$ dominates the physics. It is known that in this regime the $t-J^{\prime}$ model phase separates between an empty and half-filled phases in all dimensions 16]. This can also be understood easily by considering the atomic limit of Eq.(1), i.e., when $t \ll J_{H}, J^{\prime}$. In this case the lowest energy of a state with $N_{e} e_{g}$ electrons, including the chemical potential, is given by $-J_{H} N_{e}+J^{\prime} L-\mu N_{e}$ if $N_{e} \leq L$ and $-J_{H}\left(2 L-N_{e}\right)+J^{\prime} L-\mu N_{e}$ if $N_{e} \geq L$. The system is half-filled for $\mu=0$. When $\mu=-J_{H}\left(J_{H}\right)$ the energies of all the levels with $N_{e} \leq L\left(N_{e} \geq L\right)$ become degenerate with $E=J^{\prime} L\left(\left(J^{\prime}-2 J_{H}\right) L\right)$. For $\mu<-J_{H}\left(\mu>J_{H}\right)$ the state with the lowest energy $E=J^{\prime} L\left(\left(J^{\prime}-2 J_{H}\right) L\right)$ is the one with $N_{e}=0(2 L)$. For $-J_{H}<\mu<J_{H}$ the ground state is the state with $N_{e}=L$. Thus, at $\mu=-J_{H}\left(J_{H}\right)$ phase separation between the half-filled and the empty (fully doubly occupied) state occurs. Similar tendencies have been recently observed in one band models for manganites [17].

In Fig.3 the stable densities as a function of $J^{\prime}$ are presented. A very similar result was obtained using open boundary conditions. In the following sections the properties of the stable regions in the phase diagram will be studied.

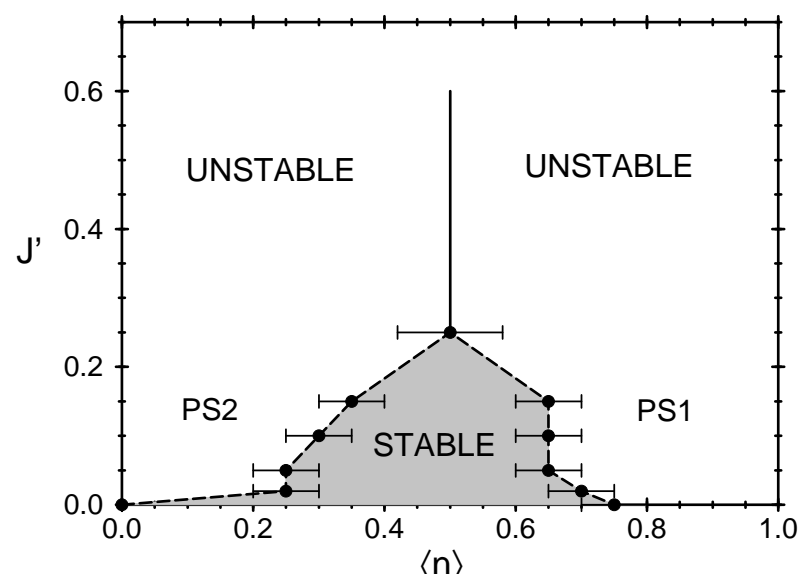

FIG. 3. The stable and unstable regions in the $J^{\prime}-\langle n\rangle$ plane are indicated for $J_{H}=8$. 


\section{MAGNETIC AND CHARGE ORDERING}

\section{A. Magnetic}

Some of the most important properties of the manganite materials are related to the magnetic order of the electrons since a remarkable interplay between ferromagnetic and antiferromagnetic phases has been observed in the experiments [2]. Actually recent neutron-scattering measurements performed on $\mathrm{La}_{2-2 \mathrm{x}} \mathrm{Sr}_{1+2 \mathrm{x}} \mathrm{Mn}_{2} \mathrm{O}_{7}$ with $x=0.4$ have reported the coexistence of short-range antiferromagnetic correlations with strong ferromagnetic fluctuations [11]. In particular, antiferromagnetic fluctuations one order of magnitude smaller in intensity than the ferromagnetic ones were observed between $100 \mathrm{~K}$ and $200 K$, i.e., above the Curie temperature. The antiferromagnetic fluctuations disappear when ferromagnetic long-range order is established.

Motivated by these experiments, in the present section we will study the behavior of the spin-spin correlation functions among the classical spins defined as

$$
\omega(r)=\frac{1}{L} \sum_{i}\left\langle\mathbf{S}_{i} \cdot \mathbf{S}_{i+r}\right\rangle,
$$

where the notation is standard. The structure factor $S(q)$ is given by the Fourier transform of the spin-spin correlations:

$$
S(q)=\sum_{r} e^{-i q r} \omega(r),
$$

where the momentum $q$ can take the values $2 \pi n / L$ with $n$ running from 0 to $L-1$. In Fig. $4, S(q)$ at $T=1 / 75$, $J^{\prime}=0.05$ and for densities $\langle n\rangle=0,0.35,0.5,0.65$ and 1 is presented. It is clearly observed in the figure that for $\langle n\rangle=0$ and 1 the structure factor peaks at momentum $q=\pi$ denoting antiferromagnetism in the system in agreement with the experimental behavior [2]. This is certainly the effect that we wanted to induce by the inclusion of a $J^{\prime}$ coupling, namely the existence of AF correlations at both ends of the $e_{g}$ density range rather than only at $x=0$. At the remaining stable values of $\langle n\rangle$ the peak occurs at $q=0$ or $q=2 \pi / 20$ signaling the presence of ferromagnetism. We observed that when the number of itinerant electrons is even and periodic boundary conditions are used, a kink appears in the ground state separating two ferromagnetic regions with opposite spin. 18 This kink causes the peak in $S(q)$ to move to $2 \pi / L$ [19]. As a result of this effect a kink appears at quarter filling when the length of the chain $L$ is a multiple of 4 . When this happens we have verified that the peak is at $q=0$ for other values of $L$ and also using open and antiperiodic boundary conditions. Then, there is no doubt that ferromagnetic correlations are dominant when the peak in $S(q)$ occurs at or close to $q=0$.

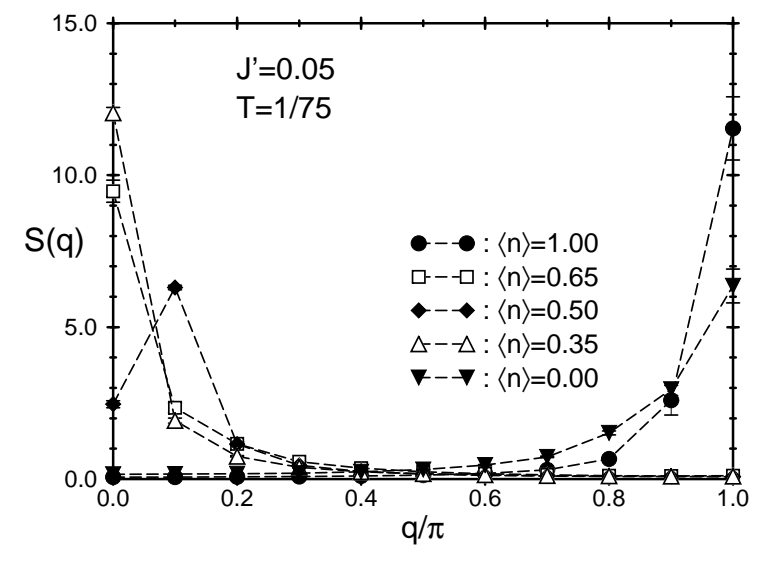

FIG. 4. Structure factor, $S(q)$, as a function of $q / \pi$ for an $L=20$ chain at $J^{\prime}=0.05$ and $T=1 / 75$. The densities are indicated in the figure.

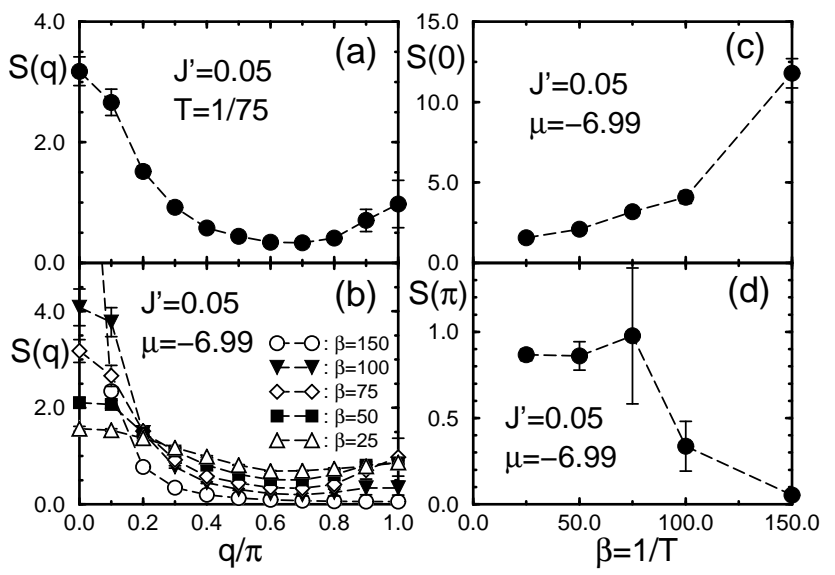

FIG. 5. (a) Structure factor as a function of $q / \pi$ on an $L=20$ chain at $T=1 / 75, \mu=-6.99$ and $J^{\prime}=0.05$. Maxima at $q=0$ and $\pi$ are clearly observed; (b) same as (a) at different temperatures indicated in the figure; (c) the structure factor at $q=0$ as a function of the inverse temperature $\beta$ for $\mu=-6.99 ;$ (d) same as (c) for $q=\pi$.

Note that for all the densities studied in Fig.4 the structure factor has a maximum at a single value of the momentum indicating that only one kind of magnetic fluctuations prevail at a given density. However, finite temperature measurements at other densities such as $\langle n\rangle=0.75$ which corresponds to an unstable density in the limit of zero temperature indicate otherwise. As it can be observed in Fig.5a, strong ferromagnetic correlations coexist with weak antiferromagnetic ones at this 
density and $T=1 / 75$. This result has similarities with the experimental data [1] discussed before, and we believe it is due to the coexistence of hole-undoped antiferromagnetic and hole-rich ferromagnetic domains rather than to a ferromagnetic spin polaron in an antiferromagnetic background, as suggested in Ref. [20]. To explore this scenario the chemical potential was fixed at $\mu=-6.99$ and $S(q)$ was measured at $T=1 / 25,1 / 50$, $1 / 75,1 / 100$ and $1 / 150$. The results are presented in Fig.5b. As the temperature is lowered the intensity of the peak at $q=0$ increases (Fig.5c) while the peak at $q=\pi$ decreases (Fig.5d). The density reaches the value 0.65 at the lowest temperature indicating that the system at 0.75 is unstable. The qualitative similarities of our results with the experimental data of Ref. [11] is clear. The relative intensity of the ferromagnetic and antiferromagnetic peaks can be tuned by properly selecting the density at finite temperature in the region that will become unstable as $T \rightarrow 0$.

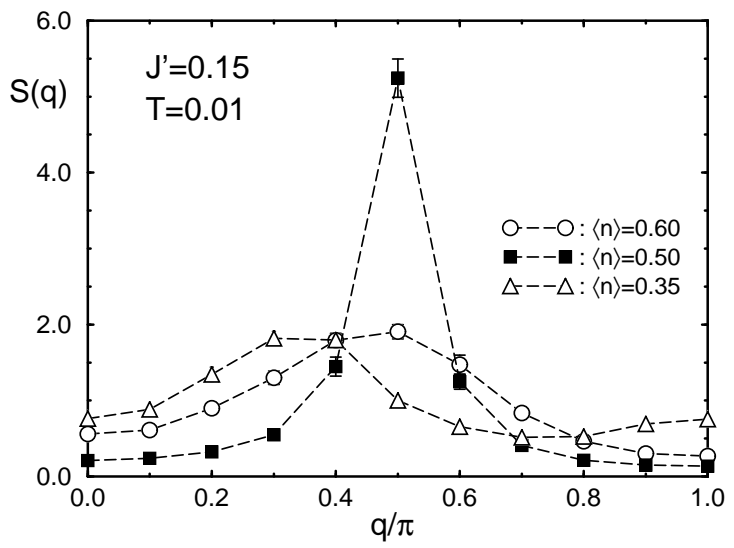

FIG. 6. Structure factor as a function of the momentum for different densities at $J^{\prime}=0.15$ and $T=0.01$.

Ferromagnetism in the low-temperature stable intermediate densities between the two regimes of phase separation PS1 and PS2 is observed up to $J^{\prime} \approx 0.1$. However, for larger values of $J^{\prime}$ weak incommensurate correlations were observed. In Fig.6, $S(q)$ at $J^{\prime}=0.15$ and for densities $\langle n\rangle=0.35,0.5$ and 0.6 is presented. A low-intensity peak at $\langle n\rangle=0.35$ and 0.6 suggests a tendency to shortrange incommensurate order with momentum $q=0.3 \pi$ and $0.5 \pi$, respectively. Note, however, that at $\langle n\rangle=0.5$ a strong peak is observed at $q=\pi / 2$. This indicates the existence of a spin twisted ground state (a spiral state with pitch $q=\pi / 2)$. The structure factor at $\langle n\rangle=0.5$ in chains of sizes ranging from $L=10$ to 30 was studied and it is presented in Fig.7a. A large increase of the peak intensity with the lattice size was not observed. However, in all the chains studied we found that the correlation length for the twisted order although finite seems fairly large as it can be observed in Fig.7b. The intensity of the incommensurate correlations at quarter-filling reaches a maximum at $J^{\prime} \approx 0.15$. As $J^{\prime}$ increases further, incommensurability at $q=\pi / 2$ weakens and at $J^{\prime} \approx 0.35$ the ground state becomes antiferromagnetic, which is the expected behavior at the stable densities in the large $J^{\prime}$ limit.
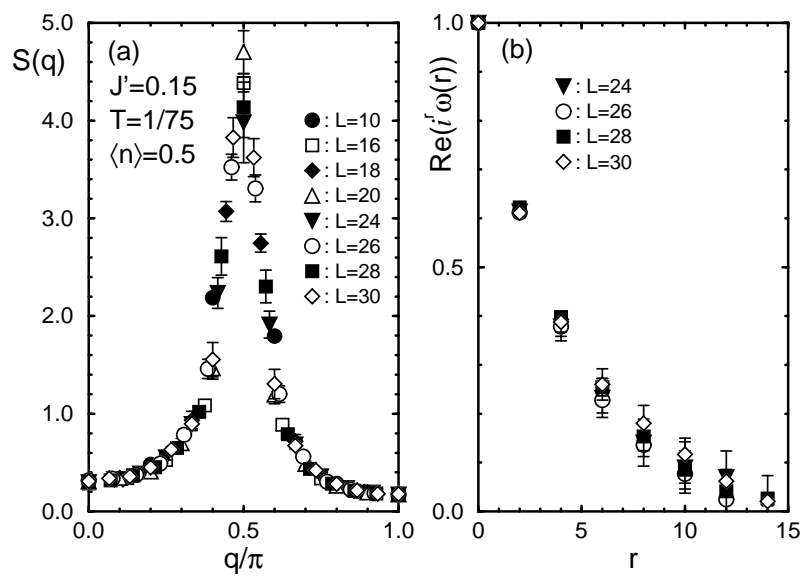

FIG. 7. (a) Structure factor as a function of $q / \pi$ at $T=1 / 75, J^{\prime}=0.15$ and $\langle n\rangle=0.5$ for $\mathrm{L}=10,16,18,20$, 24, 26, 28 and 30. A peak at $q=\pi / 2$ appears; (b) real part of the spin correlation function $\omega(r)$ multiplied by $i^{r}$ as a function of the distance $r$ for $L=24$ (triangles), 26 (circles) $L=28$ (squares) and 30 (diamonds).

\section{B. Charge}

The existence of commensurate and incommensurate charge ordered phases has been reported in the experimental literature for $\mathrm{La}_{1-\mathrm{x}} \mathrm{Ca}_{\mathrm{x}} \mathrm{MnO}_{3}$ 3]. It was observed that for values of $0.5 \leq x \leq 0.67$ antiferromagnetism coexists with commensurate charge order, and charge incommensurability was detected in a ferromagnetic phase at higher temperature. This behavior is very puzzling since ferromagnetism and charge ordering were not expected to appear together, particularly based on analysis using the double exchange model. In order to analyze the effect of the interplay of double exchange and antiferromagnetic interactions in charge ordering for the itinerant fermions, the Fourier transform $N(q)$ of the charge correlations was studied. The charge correlation functions are given by

$$
n(r)=\frac{1}{L} \sum_{i}\left\langle\left(n_{i}-\langle n\rangle\right)\left(n_{i+r}-\langle n\rangle\right)\right\rangle,
$$

where $n_{i}$ is the number operator at site $i$ for the itinerant fermions. Computationally we found that in the antiferromagnetic phase at half-filling $N(q)$ peaks at $q=\pi$ 
rather than $q=0$. This behavior is due to charge correlations that are negative (charge repulsion) at very short distances rather than to long range ordering, and it is in agreement with previous calculations [21]. In the ferromagnetic phase we observed that $N(q)$ behaves as for non-interacting spinless fermions, i.e. it reaches a maximum at $q=2 k_{F}^{s f}=2 \pi\langle n\rangle\left(k_{F}^{s f}=\pi\langle n\rangle\right.$ is the Fermi momentum for free spinless fermions) as $q$ grows from 0 , and then it becomes flat (Fig.8-a). This indicates that the value of the Hund coupling, $J_{H}=8$, is sufficiently strong for the itinerant spins to be parallel to the localized ones, behaving effectively as non-interacting spinless fermions in the charge channel.
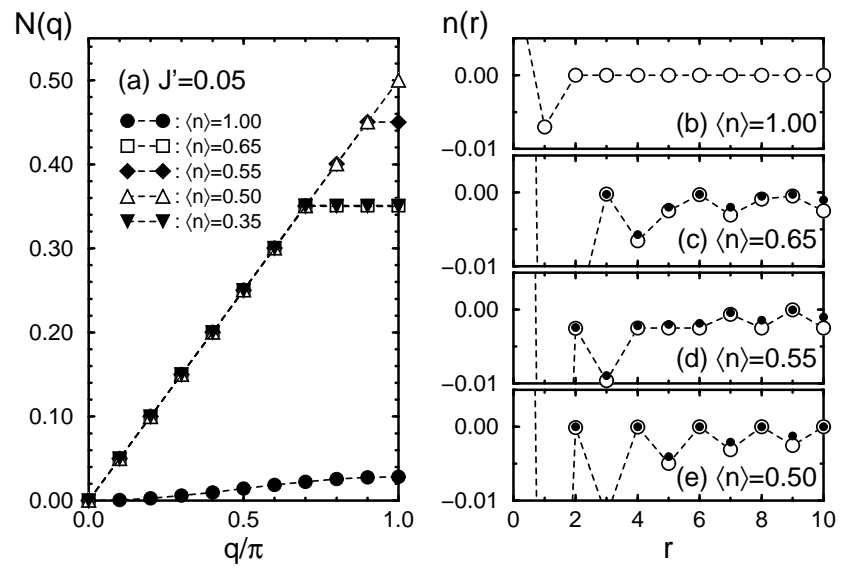

FIG. 8. (a) Fourier transform of the charge correlation functions as a function of $q / \pi$ at the densities indicated in the figure for $J^{\prime}=0.05$ on a $L=20$ chain at $T=1 / 75$; (b) Charge correlation function as a function of distance for $\langle n\rangle=1$; (c) same as (b) for $\langle n\rangle=0.65$. The small full circles are results obtained using Eq.(5); (d) same as (c) for $\langle n\rangle=0.55$; (e) same as (c) for $\langle n\rangle=0.5$.

In Fig.8b-e the charge correlations as a function of distance are presented. At half-filling (Fig.8b) the only non-negligible correlations are the nearest-neighbor ones which are negative. This effect induces a maximum at $q=\pi$ in $N(q)$. In the ferromagnetic region the charge correlations show oscillatory behavior. In particular, at quarter filling (Fig.8e) the correlations are zero at even distances and negative at odd ones. Although the system is metallic, this produces a maximum of $N(q)$ at $q=\pi$ similar to the one experimentally observed along the $a$ axis of Ca-doped manganites with $50 \%$ doping [3]. The behavior of the charge correlation for non-interacting spinless fermions is given by

$$
n(r)=\frac{-1}{2 \pi^{2} r^{2}}+\frac{\cos \left(2 k_{F}^{s f} r\right)}{2 \pi^{2} r^{2}} .
$$

This expression is strictly valid in the limit $L \rightarrow \infty$ and for $r \neq 0$ but as it can be observed from Fig.8c, $\mathrm{d}$ and e, where the correlations obtained from Eq.(5) are denoted with filled circles, they are in very good agreement with the numerical results for $L=20$. Thus, apparently this is a system that behaves like a ferromagnet in the spin channel, but is non-interacting in the charge channel.

While the qualitative behavior of $N(q)$ at half-filling is independent of $J^{\prime}$ since the ground state is always antiferromagnetic, a different behavior is observed at intermediate densities when the ground state is no longer ferromagnetic. In Fig.9a, $N(q)$ is presented for $J^{\prime}=0.15$. $N(q)$ now reaches a maximum at $q=2 k_{F}^{s f}$ and afterwards the intensity forms a plateau at a lower value. In Fig.9b-e the charge correlations as a function of the distance are shown. It is clear that the peaks in $N(q)$ are caused by oscillatory behavior of the charge correlations. It appears that a tendency to short-range charge ordering exists in the model for densities close to quarter-filling.
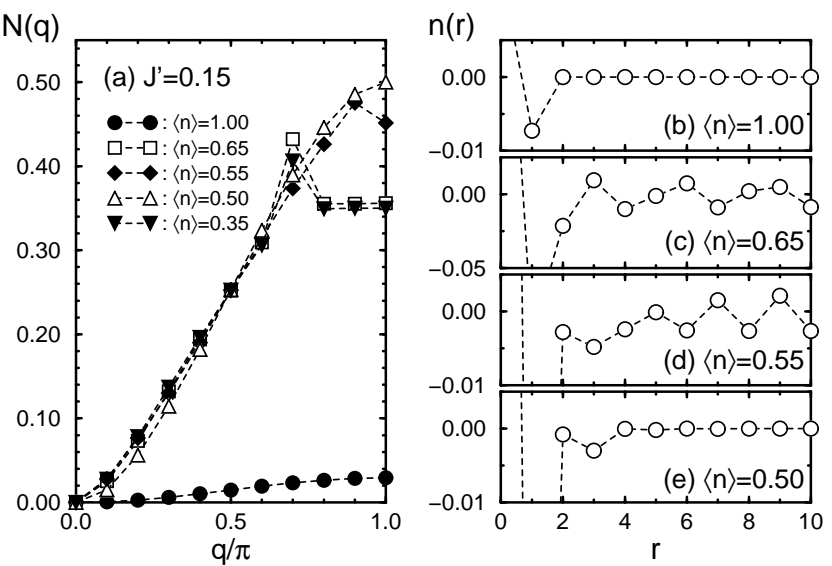

FIG. 9. (a) Fourier transform of the charge correlation functions as a function of $q / \pi$ at the densities indicated in the figure for $J^{\prime}=0.15$ on a $L=20$ chain at $T=0.01$; (b) Charge correlation function as a function of distance for $\langle n\rangle=1$; (c) same as (b) for $\langle n\rangle=0.65$; (d) same as (c) for $\langle n\rangle=0.55$; (e) same as (c) for $\langle n\rangle=0.5$.

\section{FERMI SURFACE AND SPECTRAL FUNCTIONS}

In previous sections, regions in parameter space with a variety of magnetic and charge structures were found. Here the dynamical properties of these phases will be studied. A calculation of the spectral function $A(k, \omega)$ and the study of the momentum distribution function $n(k)$ of the $e_{g}$ electrons will allow us to determine, at least qualitatively, whether a Fermi surface exists and, thus, whether the phase is insulating or metallic (this analysis will continue in the following section where the Drude weight of the optical conductivity will be discussed). In principle, the spectral function can be com- 
pared with angle-resolved photoemission (ARPES) measurements performed in $\mathrm{La}_{1.2} \mathrm{Sr}_{1.8} \mathrm{Mn}_{2} \mathrm{O}_{7}$ 22] and in $\mathrm{La}_{1-\mathrm{x}} \mathrm{Sr}_{\mathrm{x}} \mathrm{MnO}_{3}$ 23.

The spectral function $A(k, \omega)$ is defined as

$$
A(k, \omega)=-\frac{1}{\pi} \operatorname{Im} G(k, \omega),
$$

where $G(k, \omega)$ is the one-particle Green's function for the $e_{g}$ electrons. Since we are performing a Monte Carlo calculation on the classical spins only, the time-dependent Green's function can be straightforwardly calculated in real-time since the fermions do not interact among themselves directly, but only with the classical spins. The spectral functions obtained with this procedure only contain small controlled statistical errors. This should be contrasted against other calculations that use the usually uncontrolled Maximum Entropy technique.

Studying the density of states for the Hamiltonian defined in Eq.(1) we observed low-energy and high-energy bands. The separation between these two bands is regulated by the Hund coupling $J_{H}$, i.e., the lower (upper)band includes states where the spin of one of the itinerant electrons at an arbitrary site $i$ is parallel (antiparallel) to the classical spin at the same site of the lattice. The gap is about $2 J_{H}$. Each of the two bands has an internal structure and a bandwidth which are regulated in part by the antiferromagnetic coupling $J^{\prime}$.

\section{A. Half-filling}

In Fig.10a the density of states $N(\omega)$ corresponding to $J^{\prime}=0$, half-filling, and with $T=1 / 100$ on a $\mathrm{L}=20$ chain is presented. 24] The bandwidth of the upper and lower bands is $\sim 1.9 t$ and the gap separating them is $\sim 14 t$. The chemical potential lies in the gap indicating that the system is an insulator. In Fig. $10 \mathrm{~b}, A(k, \omega)$ is shown. Both below and above the chemical potential, spectral weight is observed for all of the momenta $k$, rather than only for $k$ below or above the Fermi momentum as in non-interacting electronic systems. This is reminiscent of the antiferromagnetically induced features observed at half-filling in studies of the one band Hubbard model for cuprates 25]. Below the chemical potential there is a sharp peak for $k$ smaller than $\pi / 2$ at the bottom of the lower band. The dispersion of this feature is about $t$. The corresponding opposite behavior is observed in the upper band. If $J^{\prime}$ is increased, the bandwidth of the upper and lower bands is slightly reduced due to the increase in the AF correlations, as it can be observed in Fig.11 where the half-filled density of states is presented for $J^{\prime}=0$, $0.05,0.1,0.15$ and 0.25 .
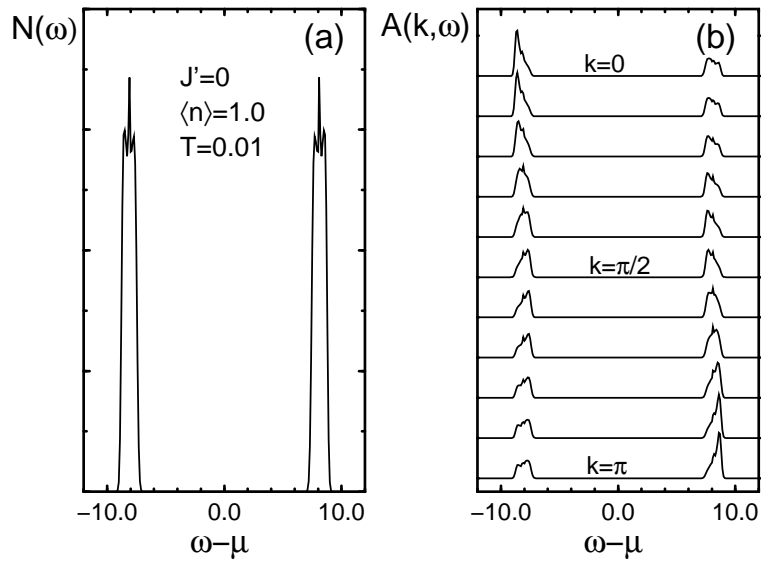

FIG. 10. (a) Density of sates $N(\omega)$ as a function of $\omega-\mu$ for $J_{H}=8, J^{\prime}=0$ at $\langle n\rangle=1$ for $L=20$ at $T=0.01$; (b) the spectral function $A(k, \omega)$ as a function of $\omega-\mu$ for the values of $k$ available on an $L=20$ lattice. The parameters are as in (a).

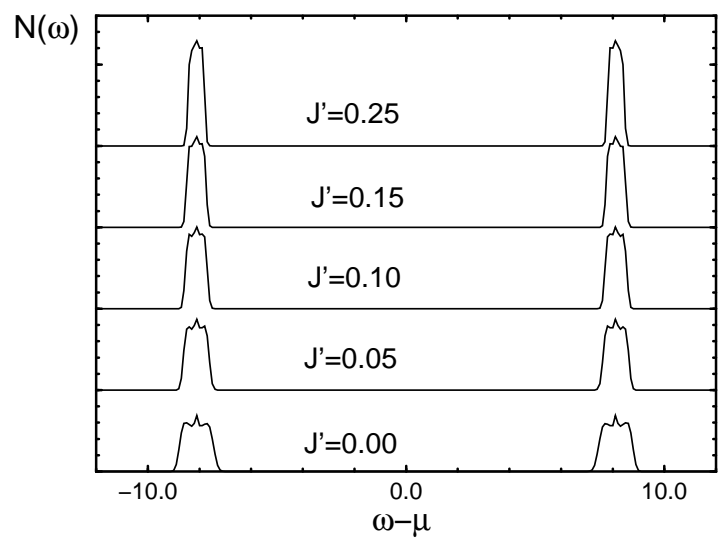

FIG. 11. Density of states as a function of $\omega-\mu$ at half-filling on $L=20$ chains at $T=0.01$ for the values of $J^{\prime}$ indicated in the figure.

\section{B. Quarter-filling}

The spectral functions change drastically in the ferromagnetic phase. First let us discuss results for density $\langle n\rangle=0.5$ (quarter-filled). $J_{H}$ still regulates the gap between the upper and lower bands. We will concentrate our attention on the lower band, since symmetrical features are observed in the upper band. In Fig.12a, $A(k, \omega)$ at $J^{\prime}=0$ is shown at quarter-filling and $T=1 / 75$ on a $L=40$ chain (similar results were obtained for shorter chains). The chemical potential lies now in the middle of the lower band indicating that the system is a metal with a Fermi surface at $k_{F}=\pi / 2$. Notice that $k_{F}$ results to be 
equal to $k_{F}^{s f}$. For each momentum $k$ there is a sharp peak which is naturally associated with a quasiparticle peak. The dispersion resembles results for a system of free spinless fermions (indicated by a dashed line in the figure), in agreement with the behavior of the charge correlations described in the previous section. The bandwidth is $4 t$. The density of states can be seen in Fig.12b. It resembles $N(\omega)$ for spinless fermions at half-filling which for a finite length chain typically presents several spikes that become a smooth function only for large enough chains. Thus, the spikes observed in Fig.12b near the chemical potential are likely finite size effects, and the quasiparticle peak crosses the chemical potential as in a standard metal. Similar behavior is observed for $J^{\prime}$ up to approximately 0.11 in the ferromagnetic phase.
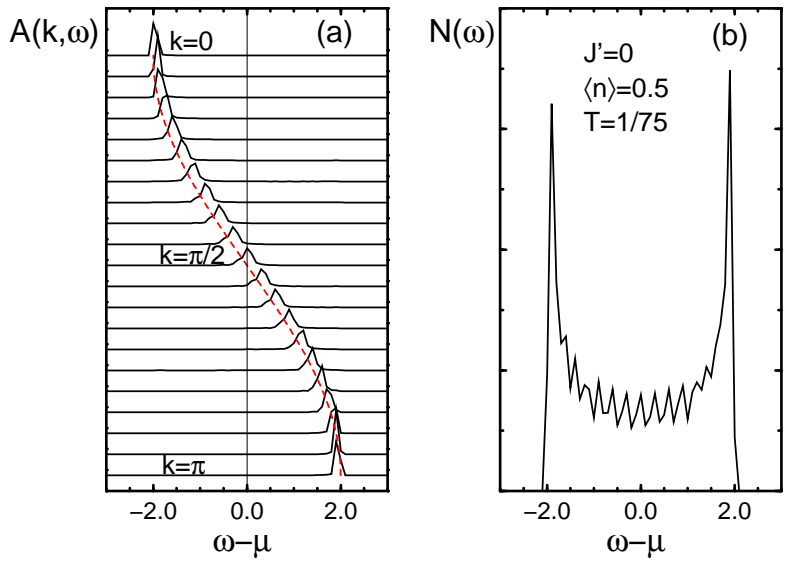

FIG. 12. (a) The spectral function $A(k, \omega)$ as a function of $\omega-\mu$ for the values of $k$ available on an $L=40$ lattice for $J_{H}=8, J^{\prime}=0, T=1 / 75$ at $\langle n\rangle=0.5$. The dashed line is the dispersion for spinless fermions; (b) Density of states as a function of $\omega-\mu$. The parameters are as in (a).

However, as $J^{\prime}$ increases and the incommensurate phase is reached the behavior of the spectral function at quarter-filling changes dramatically. Results for $J^{\prime}=$ 0.15 are presented in Fig.13 (remember that for this value of $J^{\prime}$ strong incommensurate spin correlations were detected in the study of $S(q))$. A gap has now opened and the chemical potential lies in it indicating that the system became an insulator. As shown in Fig.13-b the size of the gap appears to be independent of the size of the chains. Fig.13-a shows that considerable spectral weight appears above and below the chemical potential for momenta close to the Fermi momentum $k_{F}^{s f}=\pi / 2$. The dispersion for the features above and below the chemical potential in the lower band is of the order of 2.5t. With increasing $J^{\prime}$ the system remains insulating, but it was observed that the size of the gap decreases while antiferromagnetic correlations increase. The gap closes up for $J^{\prime} \approx 0.6$ when the quarter-filled state is no longer stable. In Fig.14 the density of states is presented for different values of $J^{\prime}$ at $\langle n\rangle=0.5$ using $L=20$ chains. Once again, the spiky structure for $J^{\prime}=0.1$ or smaller is a finite size effect that disappears as the size of the lattice grows. However, the gap observed for $J^{\prime} \geq 0.11$ is not spurious and signals an insulating regime. The opening of this gap is not just a one dimensional effect. In Fig. 15 we present the density of states at quarter-filling for $J_{H}=8$ and $J^{\prime}=0.25$ at $T=1 / 50$ on a $4 \times 4$ and a $6 \times 6$ lattice with $\mathrm{PBC}$. It can be seen that a gap has opened in the lower band and the chemical potential lies in it, denoting insulating behavior. The finite size effects do not seem strong.
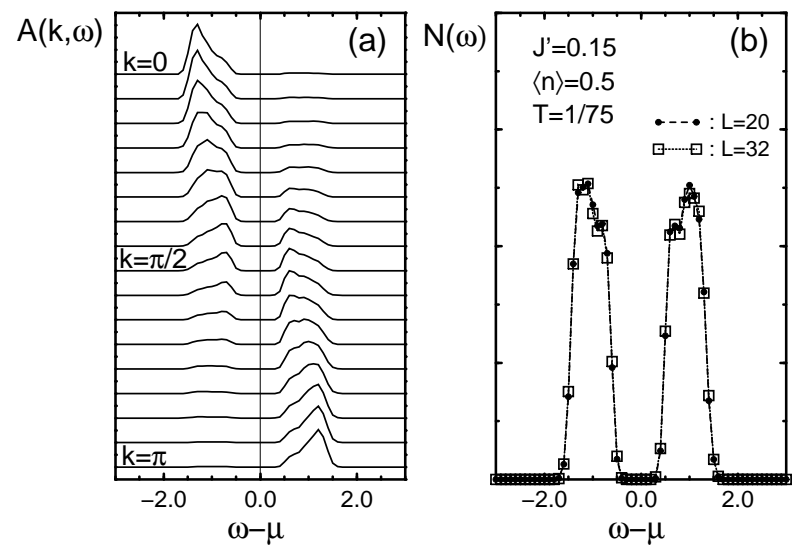

FIG. 13. (a) The spectral function $A(k, \omega)$ as a function of $\omega-\mu$ for the values of $k$ available on an $L=20$ lattice for $J_{H}=8, J^{\prime}=0.15, T=1 / 75$ at $\langle n\rangle=0.5$; (b) Density of states as a function of $\omega-\mu$. The parameters are as in (a) but $L=20$ and 32 .

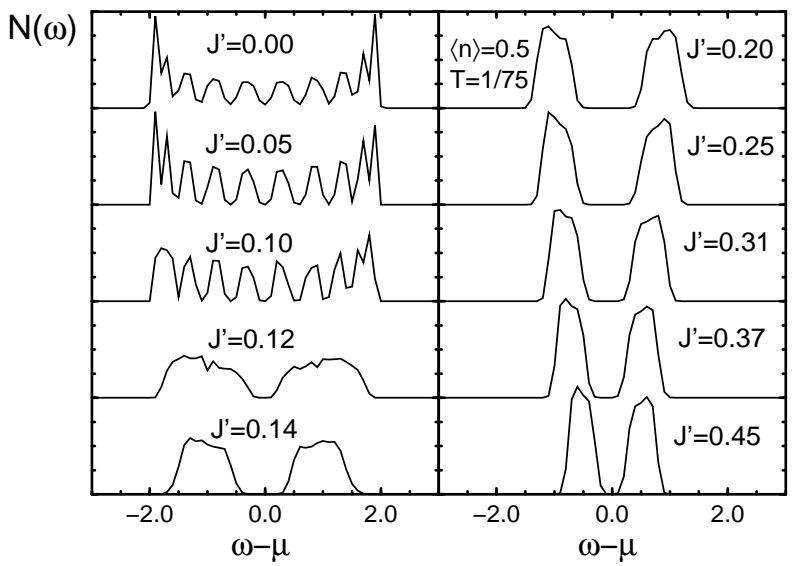

FIG. 14. Density of states as a function of $\omega-\mu$ for different values of $J^{\prime}$ at quarter filling for $L=20$ and $T=1 / 75$. 


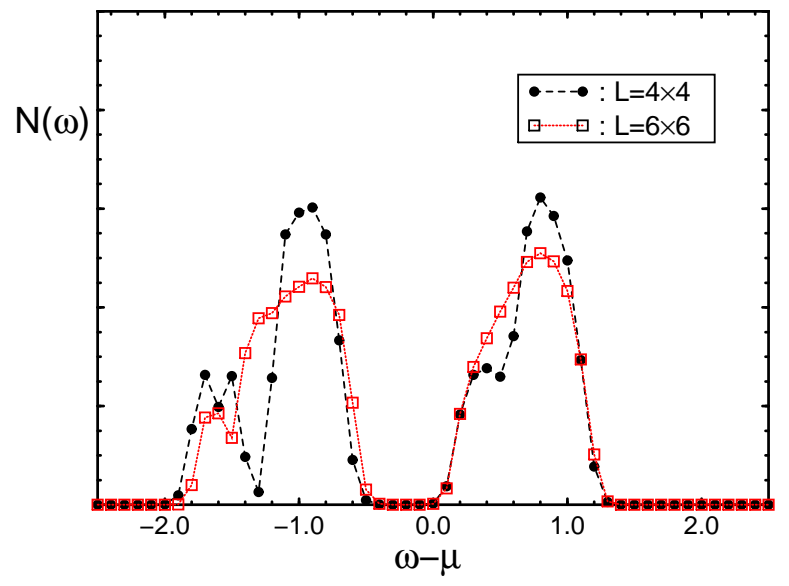

FIG. 15. Density of states as function of $\omega-\mu$ for $J^{\prime}=0.25$ at quarter filling for $4 \times 4$ and $6 \times 6$ lattices at $T=1 / 50$ with PBC.

\section{Other Densities}

The spectral functions at densities below and above 0.5 were also studied. In the ferromagnetic region a behavior resembling free spinless fermions was observed, as it is shown in Fig.16 where $A(k, \omega)$ for $J^{\prime}=0.1$ is presented at (a) $\langle n\rangle=0.55$ and (b) $\langle n\rangle=0.45$. As pointed out in Sec.IV.B, in the charge channel the itinerant electrons behave as free spinless fermions with $k_{F}^{s f}=\pi\langle n\rangle$. In the insulating phase, on the other hand, the density of states changes qualitatively away from quarter filling. When $N_{e}$ electrons ( $N_{h}$ holes) are introduced in the quarter filled state, spectral weight is redistributed so that a new peak develops inside the gap and, thus, a three peak structure is observed. The spectral weight in the original two peaks changes from $L / 2$ to $L / 2-N_{e}\left(L / 2-N_{h}\right)$ while the spectral weight of the new structure is given by $2 N_{e}\left(2 N_{h}\right)$. [26] However, the chemical potential still lies in the gap indicating that the system continues to be an insulator as it can be seen in Fig.17 where the density of states for $J^{\prime}=0.15$ at different values of $\langle n\rangle$ is presented. For the cases of $\langle n\rangle=0.55$ and 0.45 the spectral function is shown in Fig.18. It can clearly be seen that, for all values of the momentum $k$, spectral weight is being transferred to the middle of the gap. Notice that while the two external features disperse as a function of the momentum $k$, the peak at the center does not. This third peak is located exactly in the middle of the gap and it indicates that as electrons or holes are added to the quarter filled insulating system, localized states form in the middle of the gap.

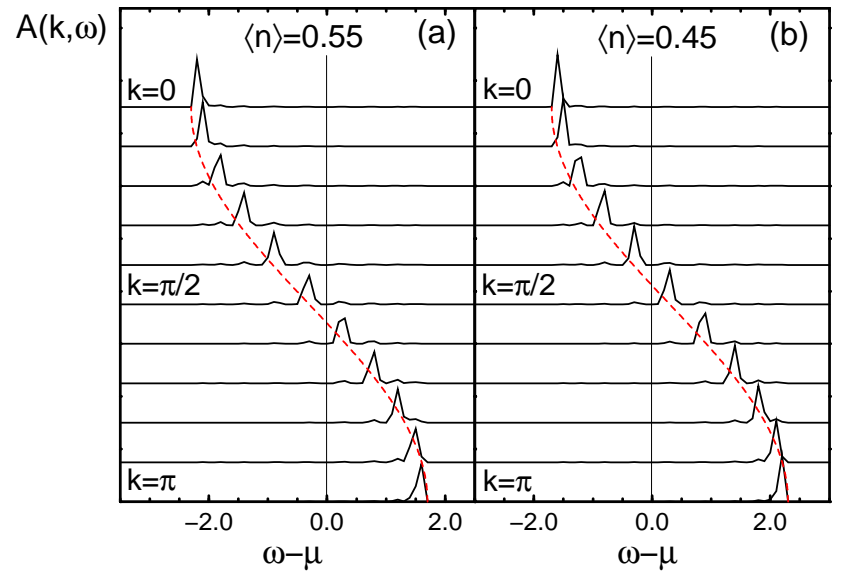

FIG. 16. (a) The spectral function $A(k, \omega)$ as a function of $\omega-\mu$ for the values of $k$ available on an $L=20$ lattice for $J_{H}=8, J^{\prime}=0.1, T=1 / 100$ at $\langle n\rangle=0.55$; (b) same as (a) for $\langle n\rangle=0.45$. The dashed line is the dispersion for spinless fermions.

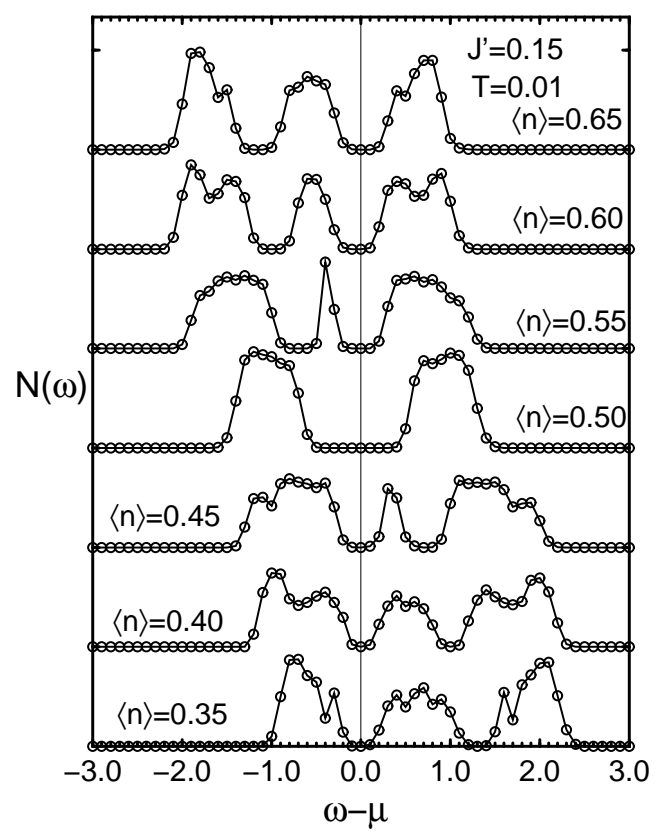

FIG. 17. Density of states $N(\omega)$ as a function of $\omega-\mu$ for $J_{H}=8, J^{\prime}=0.15$ at different densities for $L=20$ at $T=0.01$. 


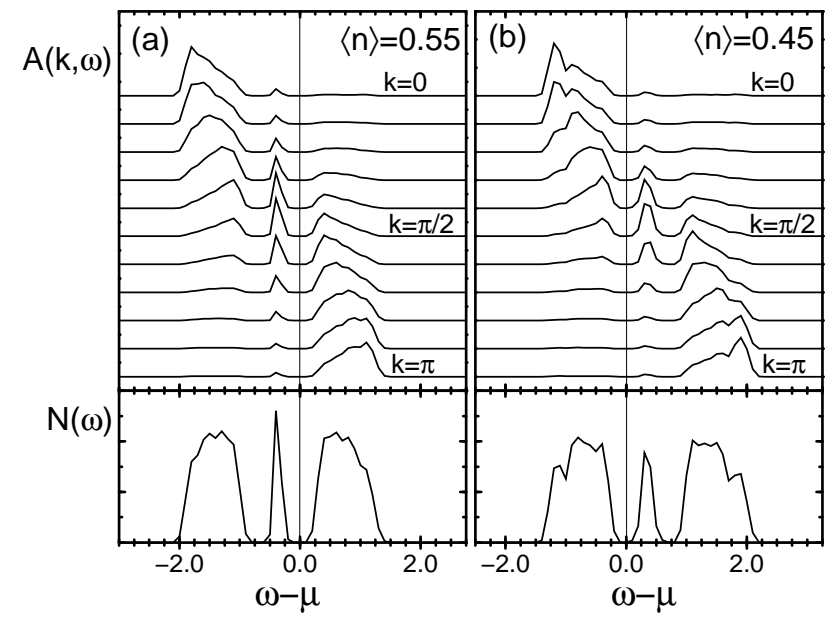

FIG. 18. (a) The spectral function $A(k, \omega)$ and the density of states $N(\omega)$ as a function of $\omega-\mu$ on an $L=20$ lattice for $J_{H}=8, J^{\prime}=0.15$ at $\langle n\rangle=0.55$ at $T=0.01$; (b) same as (a) for $\langle n\rangle=0.45$.

The occupation number $n(k)$ can be calculated directly from the Green's functions or just by integrating $A(k, \omega)$ on $\omega$ up to the chemical potential. In Fig.19 $n(k)$ for quarter filling and different values of $J^{\prime}$ is presented. A sharp change in $n(k)$ suggests the existence of a Fermi surface while a smooth behavior is more compatible with the opening of a gap. The change of behavior between $J^{\prime}=0.10$ and 0.15 is very clear. The analysis of this curves complements the previous study of the spectral functions. Analogous results were obtained in both cases.

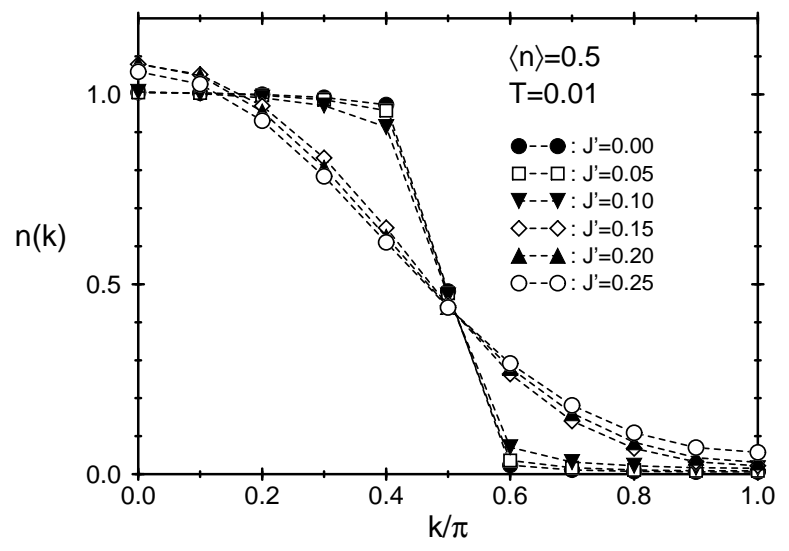

FIG. 19. $n(k)$ as a function of $k$ for different values of $J^{\prime}$ for $T=0.01$ at quarter filling on an $L=20$ chain.

Some experimental ARPES studies for manganites have found a strong dependence of the density of states with the temperature 222]. In particular the appearance of a pseudogap with increasing temperature was remarked. In Fig.20 the density of states at quarter-filling for $J^{\prime}=0$ is presented at different temperatures. As the temperature increases the peaks due to finite size effects are smeared out but the density of states retains its qualitative characteristics. There are no indications of a pseudogap developing as the temperature increases. Thus, it appears that some experimental observations may be due to effects that go beyond the electronic interactions considered in this work. Probably more than one orbital per site as well as phonons are needed to account for this nontrivial effect.

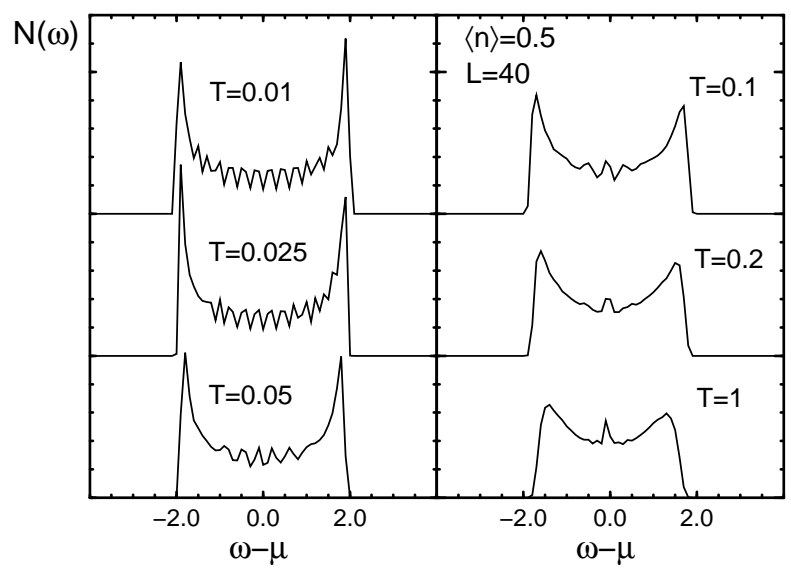

FIG. 20. The density of states as a function of $\omega-\mu$ at different temperatures for $J_{H}=8, J^{\prime}=0$ at quarter filling on a 40 site chain.

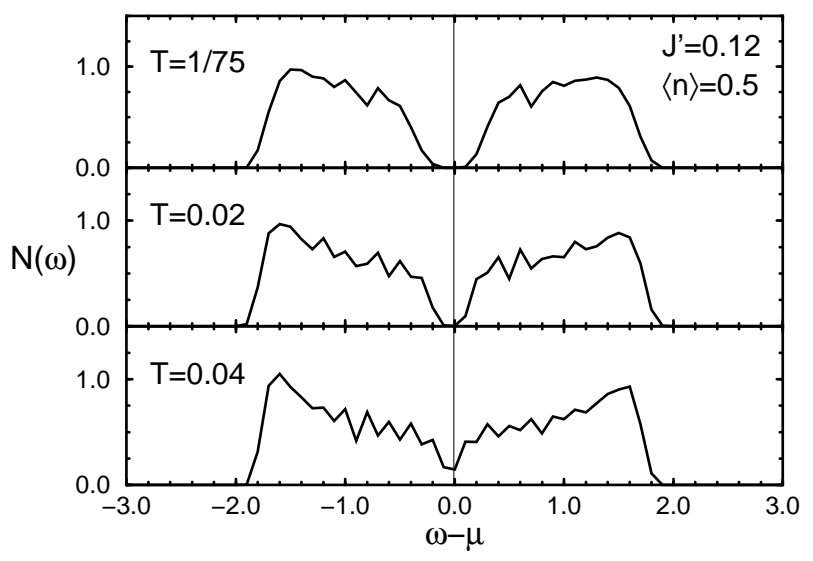

FIG. 21. The density of states as a function of $\omega-\mu$ at different temperatures for $J_{H}=8, J^{\prime}=0.12$ at quarter filling on a 22 site chain. 
For completeness, the temperature dependence of the density of states was also studied at quarter-filling in the insulating phase. In Fig.21, where results for $J^{\prime}=0.12$ are presented, it can be seen that an increase in temperature closes the gap in the density of states transforming it into a pseudogap. These results suggest that the real manganite materials could be located right at the border between the metallic and insulating regimes reported here.

\section{OPTICAL CONDUCTIVITY AND DRUDE WEIGHT}

A very important characteristic of the manganite materials is the large change in resistivity observed experimentally with small changes in doping, temperature, or magnetic fields. In addition, experimental measurements of the optical conductivity in $\mathrm{La}_{1-\mathrm{x}} \mathrm{Sr}_{\mathrm{x}} \mathrm{MnO}_{3}$ with $0 \leq x \leq 0.3$ [27] indicate the existence of a gap in the optical spectrum at half-filling which becomes a pseudogap in the ferromagnetic region at high temperatures. This behavior has similarities with those observed in ARPES data. To study the extent to which electronic interactions can account for the optical measurements, here we have calculated the optical conductivity defined as:

$$
\sigma(\omega)=\frac{\pi\left(1-e^{-\beta \omega}\right)}{\omega L} \times \int_{-\infty}^{\infty} \frac{d t}{2 \pi} e^{i \omega t}\left\langle j_{x}(t) j_{x}(0)\right\rangle
$$

where $j_{x}$ is the current operator in the $x$-direction given by

$$
j_{x}=i t e \sum_{j, s}\left(c_{j+\hat{x}, s}^{\dagger} c_{j, s}-h . c .\right)
$$

with the rest of the notation standard. At $\omega=0$ there is a Drude weight with an intensity $D$ given by:

$$
\frac{D}{2 \pi e^{2}}=\frac{<-\hat{T}>}{4 L}-\frac{1}{2 \pi e^{2}} \int_{0^{+}}^{\infty} d \omega \sigma(\omega)
$$

where $\hat{T}$ is the kinetic energy operator and $e$ is the electronic charge. Hereafter we set $e=1$.

In Fig.22a the Drude weight at density $\langle n\rangle=0.5$ is presented as a function of $J^{\prime}$ for chains of different lengths, and at low temperature. The results suggest that the Drude weight of the system decreases as $J^{\prime}$ increases and it actually vanishes for $J^{\prime} \approx 0.11$, i.e., where the metalinsulator transition was observed in previous sections by monitoring the spectral function and $n(k)$. The effect of the temperature on the optical conductivity was also studied. In Fig.22b the Drude weight is plotted as a function of temperature for several values of the antiferromagnetic coupling $J^{\prime}$ working at quarter-filling. For values of $J^{\prime}$ that lead to a ferromagnetic ground state at low temperature, the Drude weight increases as the temperature decreases. On the other hand, a decrease in the
Drude weight with decreasing temperatures is observed when the ground state at low temperature is an insulator with strong spin-incommensurate correlations.
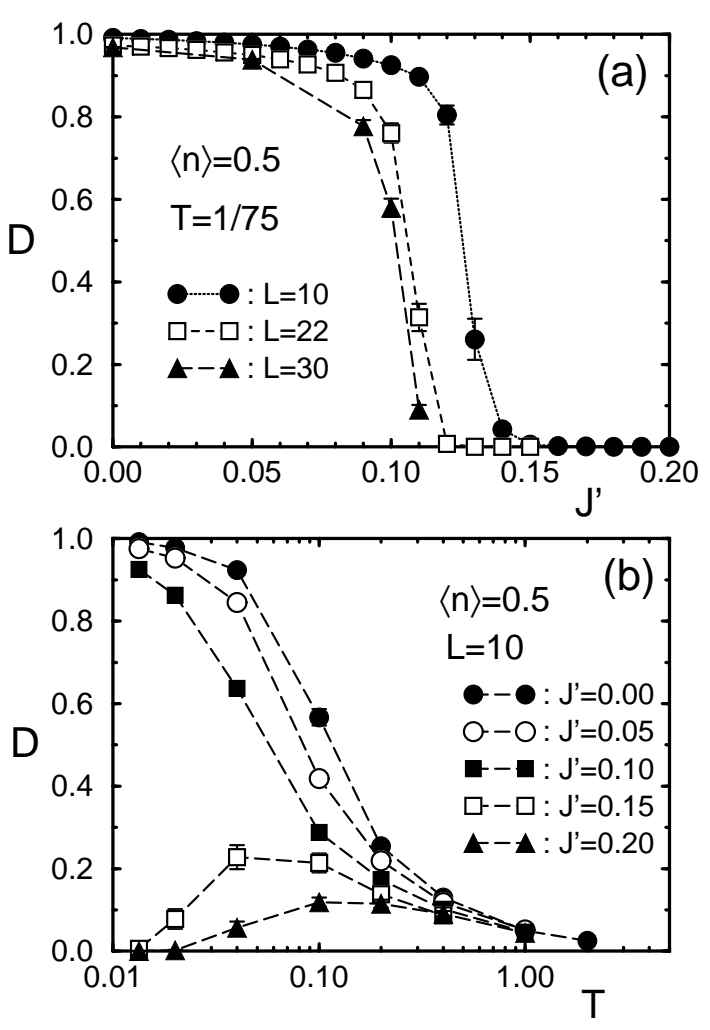

FIG. 22. (a) The Drude weight for $\langle n\rangle=0.5$ as a function of $J^{\prime}$ for chains of different lengths; (b) Drude weight as a function of temperature at quarter filling. The values of $J^{\prime}$ are indicated in the figure.

In Fig.23 the optical conductivity for $J^{\prime}=0$ at different temperatures is presented. It is clear that the spectral weight on the high-energy side, i.e. across the $2 J_{H}$ gap, decreases as the temperature decreases while the spectral weight on the low lying band increases. This occurs because at high temperature both the spins up and down have similar occupations of their lower bands and the optical gap transitions have contributions from both kind of spins. As the temperature is lowered and ferromagnetism develops, the density of states for the spins antiparallel to the classical spins goes up in energy while the density of states for the parallel spins at low energy increases providing low energy states for transitions, and as a consequence the transitions across the $2 J_{H}$ gap diminish. Similar results have been obtained in infinite dimension. 28. This behavior is in qualitative agreement with experimental measurements of the optical conductivity in doped $\mathrm{LaMnO}_{3}$ 27. 

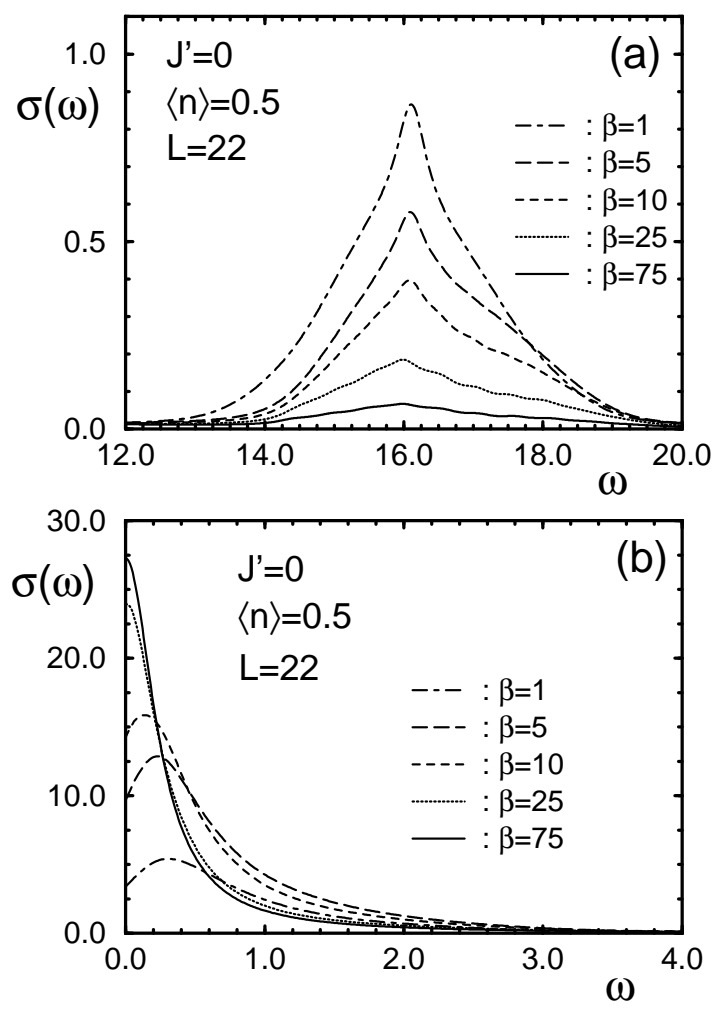

FIG. 23. Optical conductivity as a function of $\omega$ for $J_{H}=8, J^{\prime}=0$ and quarter filling for different values of the temperature; (a) high energy; (b) low energy. A lorentzian with width 0.25 was used.

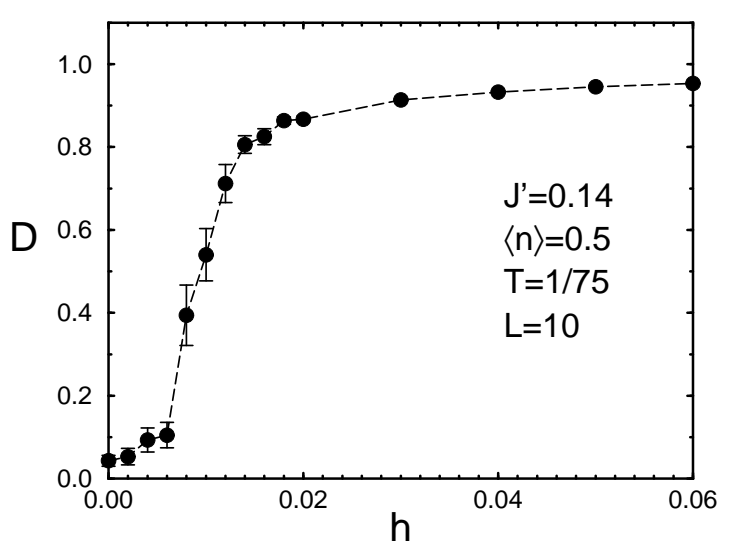

FIG. 24. Drude weight as a function of magnetic field for $J^{\prime}=0.14, T=1 / 75$ at quarter filling on an $L=10$ chain.

Finally, for completeness the influence of a magnetic field on our results was also considered. The external magnetic field was coupled to the itin- erant and localized electrons via a Zeeman term, $-h \sum_{i}\left(\sum_{\alpha, \beta} c_{i, \alpha}^{\dagger}\left(\sigma_{z}\right)_{\alpha, \beta} c_{i, \beta}+3 S_{i}^{z} / 2\right)$. A insulator-metal transition induced by the magnetic field $h$ was observed for values of $J^{\prime}$ where the ground state is an insulator, as it can be seen in Fig.24.

\section{PHASE DIAGRAM}

The information about the Kondo Hamiltonian presented in the previous sections allows us to obtain the low temperature phase diagram as a function of the antiferromagnetic coupling $J^{\prime}$ and the density $\langle n\rangle$ in the limit of large Hund coupling. The full phase diagram is presented in Fig.25. For $J^{\prime}$ smaller than $\sim 0.11$ the ground state is a ferromagnetic metal for $\langle n\rangle$ between $\sim 0.25$ and $\sim 0.65$ and an antiferromagnetic insulator for the empty and half-filled systems that are phase separated from the intermediate densities. This is the region of the phase diagram which is relevant for comparisons with experimental results for the manganites. When $J^{\prime}$ becomes larger than $\sim 0.11$ a metal-insulator transition occurs and the system becomes an incommensurate insulator at intermediate fillings. In particular the ground state at quarter-filling appears to be a spin spiral state with a maximum at $q=\pi / 2$ in the magnetic structure factor, and finite but very long correlation lengths. The allowed intermediate densities diminish as $J^{\prime}$ increases and for $J^{\prime} \approx 0.25$ only quarter-filling is stable. At $J^{\prime} \approx 0.35$ the quarter filled ground state becomes antiferromagnetic and for $J^{\prime}$ larger than $\sim 0.6$ only the half-filled and the empty phases are stable. Both of them correspond to antiferromagnetic insulators.

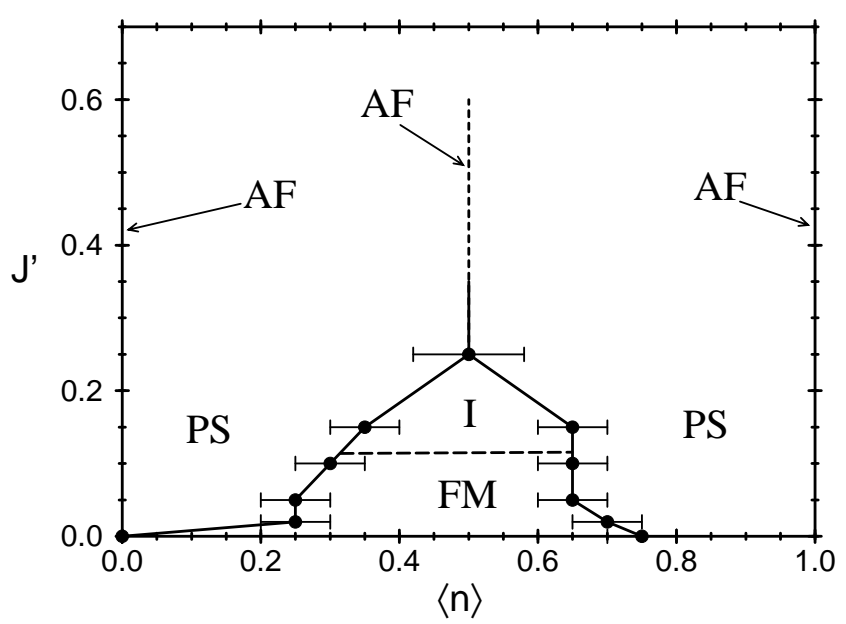

FIG. 25. The phase diagram for the Kondo lattice Hamiltonian with ferromagnetic Hund's coupling $J_{H}=8$. AF indicates the antiferromagnetic phases, I the insulating region, FM the ferromagnetic phase and PS denotes phase separation. 


\section{SUMMARY}

In this paper we have studied a ferromagnetic Kondo lattice model with classical localized spins coupled antiferromagnetically, using Monte Carlo methods. Working at large Hund coupling we found a very rich phase diagram as the density of itinerant electrons and the strength of the antiferromagnetic coupling $J^{\prime}$ was changed. In particular, phase separation between holeundoped antiferromagnetic and hole-rich ferromagnetic states was observed at high electronic densities. Reciprocally, phase separation between electron-undoped antiferromagnetic and electron-rich ferromagnetic regions was observed in the limit of low $e_{g}$ electronic density. At intermediate densities, we found a metallic ferromagnetic phase for small $J^{\prime}$ separated from an insulating regime with a transition located at $J^{\prime} \approx 0.11$. The insulating phase has very strong incommensurate magnetic correlations with a structure factor that peaks at $q=\pi / 2$ for quarter-filling. At $J^{\prime} \approx 0.35$ the quarter-filled ground state becomes antiferromagnetic and beyond $J^{\prime} \approx 0.6$ only the half-filled and empty antiferromagnetic phases are stable.

$J^{\prime}=0.05$ is possibly a good value to compare with experimental results. We have observed ferromagnetic and antiferromagnetic spin correlations coexisting for $\langle n\rangle=0.75$ at $T=1 / 75$. As in neutron scattering experiments the ferromagnetic peak increases as the temperature decreases, while the antiferromagnetic excitations vanish in the ferromagnetic phase. In the present model this behavior is due to the coexistence of ferromagnetic and antiferromagnetic domains at high temperature, which are a consequence of the tendency of the system to phase separate [10].

The study of dynamical properties shows the existence of interesting metal-insulator transitions as a function of temperature and $J^{\prime}$. A transference of spectral weight from higher to lower energies with decreasing temperature, in agreement with experimental results, is observed in the optical conductivity. The metal-insulator transition with increasing $J^{\prime}$ is a feature of the model also present in two dimensions and it is very likely that the transition will also occur in three dimensions.

Summarizing, the present results show that several properties of the manganites, such as the change in magnetic ordering as a function of concentration and transference of spectral weight from high to low energies in the optical conductivity with temperature, may be attributed principally to simple electronic interactions. However, additional factors will have to be considered to explain, for example, the opening of gaps in the density of states with increasing temperature. The addition of phononic degrees of freedom will probably be relevant and we will take them into consideration in future work.

\section{ACKNOWLEDGEMENTS}

We would like to acknowledge useful comments by $\mathrm{N}$. Furukawa, E. Dagotto and G. Aeppli. S.Y. is supported by the Japanese Society for the Promotion of Science. A.M. is supported by NSF under grant DMR-95-20776. Additional support is provided by the National High Magnetic Field Lab and MARTECH.

[1] S. Jin et al., Science 264,413 (1994).

[2] P. E. Schiffer et al., Phys. Rev. Lett.75, 3336 (1995).

[3] C. H. Chen and S-W. Cheong, Phys. Rev. Lett.76, 4042 (1996).

[4] R. A. Moham Ram et al., J. Solid St. Chem.70, 82 (1987).

[5] C. Zener, Phys. Rev.82, 403 (1951).

[6] P. W. Anderson and H. Hasegawa, Phys. Rev.100, 675 (1955).

[7] P. -G. de Gennes, Phys. Rev.118, 141 (1960).

[8] A.J. Millis et al., Phys. Rev. Lett.74, 5144 (1995).

[9] S. Ishihara, J. Inoue and S. Maekawa, Phys. Rev. B 55, 8280 (1997).

[10] S. Yunoki, J. Hu, A. Malvezzi, A. Moreo, N. Furukawa and E. Dagotto , preprint cond-mat/9706014 to appear in Phys. Rev. Lett.; E. Dagotto, S. Yunoki, A. Malvezzi, A. Moreo, J. Hu, S. Capponi, D. Poilblanc and N. Furukawa, preprint cond-mat/9709029.

[11] T.G. Perring, G. Aeppli, Y. Moritomo and Y. Tokura, Phys. Rev. Lett. 78, 3197 (1997).

[12] K. Kubo and N. Ohata, J. Phys. Soc. Jap33, 21 (1972).

[13] N. Furukawa, J. Phys. Soc. Jap63, 3214 (1994).

[14] Some authors have attempted to simplify the Hamiltonian by replacing the spins $3 / 2$ by spins 1 or $1 / 2$ but the quantum effects associated to small spins are very strong. For this reason our approach is to replace the spins $3 / 2$ by classical ones which we believe capture better their physics than smaller spins.

[15] We made additional runs at $T=1 / 125$.

[16] E. Dagotto, Rev. Mod. Phys. 66, 763 (1994).

[17] J. Riera, K. Hallberg, and E. Dagotto, Phys. Rev. Lett. 79, 713 (1997).

[18] K. Kubo, J. Phys. Soc. Jpn., 51, 782 (1982); J. Zhang, H. Röder, A. R. Bishop and S. A. Trugman, J. Phys. Condens. Matter, 9, L157 (1997).

[19] If antiperiodic BC are used $S(q)$ peaks at $q=0$ in the ferromagnetic phase. However, in this case $q=\pi / 2$, which will be relevant later in this work, is not allowed in the lattice.

[20] P. Horsch, J. Jaklic and F. Mack, preprint condmat/9708007.

[21] Y.C. Chen et al., Phys. Rev. B 50, 655 (1994).

[22] D.S. Dessau et al., preprint.

[23] T. Saitoh et al., Phys. Rev. B 51, 13942 (1995).

[24] $\Delta \omega=0.1$. The same value was used in all the figures where spectral data are displayed. 
[25] S. Haas, A. Moreo, and E. Dagotto, Phys. Rev. Lett. 74, 4281 (1995), and references therein.

[26] The total spectral weight is $2 L$.

[27] Y. Okimoto et al., Phys. Rev. Lett. 75, 109 (1995) and Phys. Rev. B 55, 4206 (1997).

[28] N. Furukawa, J. Phys. Soc. Jap. 64, 3164 (1995). 